\title{
检测一氧化碳分子苂光探针的研究进展
}

\author{
魏 超* 张平竹李小六* \\ (河北大学化学与环境科学学院 河北省化学生物学重点实验室 药物化学与分子诊断教育部 \\ 重点实验室 河北保定 071002)
}

\begin{abstract}
摘要 一氧化碳 $(\mathrm{CO})$ 是一种重要的内源性气体递质分子, 参与调节生命体的多种生理和病理过程. 因此, 选择性识别 和高灵敏检测生物体内源 $\mathrm{CO}$ 具有十分重要的生物学和医学意义. 苂光探针法具有选择性好、灵敏度高、适于高通量 節选, 尤其是对生物样品无侵入性损伤, 以及可实现实时原位检测等优势, 因此, 利用苂光探针技术检测细胞、组织和 活体内 $\mathrm{CO}$ 浓度的变化是近年来研究热点之一. 综述了近十年来 $\mathrm{CO}$ 苂光探针的研究进展, 概述了相关荧光探针的设计 理念、检测机理及生物应用, 探讨了探针的结构和性能之间的关系, 展望了 CO 荧光探针的发展趋势和应用前景. 关键词 一氧化碳; 气体递质; 荧光探针; 荧光成像
\end{abstract}

\section{Progress in Fluorescent Probes for Carbon Monoxide Detecting}

\author{
Wei, Chao* Zhang, Pingzhu Li, Xiaoliu* \\ (Key Laboratory of Chemical Biology of Hebei Province, Key Laboratory of Medicinal Chemistry and Molecular \\ Diagnosis of Ministry of Education, College of Chemistry and Environmental Science, \\ Hebei University, Baoding, Hebei 071002)
}

\begin{abstract}
As an important endogenous gas transmitter, carbon monoxide (CO) has been found taking part in multiple physiological and pathological processes for life. Therefore, the selective recognition and sensitive detection of CO are of great biological and medical significance. Fluorescent probe method is highlighted by its good selectivity, high sensitivity, suitable for high-throughput screening, especially noninvasive detection, and real-time monitoring in situ. Therefore, the development of fluorescent probes for intracellular $\mathrm{CO}$ has been becoming one of the hot topics. Herein, the progress during the last decade of fluorescent molecular probes based on the small molecules for $\mathrm{CO}$ detection is reviewed. These fluorescent probes are classified and concluded according to the design concepts, detection mechanism, and biological applications. In addition, the relationship between molecular structures and properties is elucidated. Finally, the challenge and application prospects for the development of $\mathrm{CO}$ fluorescent probes are also discussed.
\end{abstract}

Keywords carbon monoxide; gasotransmitter; fluorescent probe; fluorescent imaging

一氧化碳 $(\mathrm{CO})$ 是一种无色无味的气体分子, 空气中 $\mathrm{CO}$ 主要由含碳物质不完全燃烧产生. CO 可以与血红蛋 白结合, 并且结合强度约为氧气的 200 倍. 当环境中 CO 浓度过高时，可通过竞争性结合血红蛋白，降低血氧浓 度, 造成生命体昏厥、甚至死亡. 因此, $\mathrm{CO}$ 被称为危害 人类的 “隐形杀手”. 近年来研究发现, $\mathrm{CO}$ 是植物和动 物体内一种内源性气体信号分子, 在维系动植物生命体 正常运行过程中扮演者重要的角色 ${ }^{[1]}$. 动植物内源性 $\mathrm{CO}$ 均主要由血红素氧合酶(HO)催化分解亚铁血红素 (heme) 产生 ${ }^{[2,3]}$. 植物内源性 CO 可以调节植物生长发育,
如对逆境(如高盐、重金属、紫外线、活性氧等)响应、 气孔运动、根系发育, 以及与其它信号分子的相互作用 等 ${ }^{\left[{ }^{4} 8\right]}$. 动物内源性 $\mathrm{CO}$ 可以激活鸟苷酸环化酶活性、参 与呼吸节律调节、缓解氧化应激、调节胰岛素释放和降 低血压等 ${ }^{[\rho 11]}$. 此外，相关研究发现内源性 CO 浓度异 常与多种疾病的发生发展密切相关, 包括神经退行性病 变、心血管疾病、肺病、糖尿病、肥胖症、败血症和癌 症等 ${ }^{[12 \sim 14]}$. 因此, 开发 “可视化” 内源 CO 的检测方法, 是 $\mathrm{CO}$ 生物学功能和疾病诊断等相关研究发展迫切需要 解决的问题之一.

\footnotetext{
* Corresponding authors. E-mail: weichao@hbu.edu.cn; lixl@hbu.cn.

Received June 24, 2019; revised July 18, 2019; published online August 7, 2019

Project supported by the National Natural Science Foundation of China (No. 21778013), the Natural Science Foundation of Hebei Provience (No. B2018201234), and the Colleges and Universities Science Technology Research Project of Hebei Province (No. QN2017015).

国家自然科学基金(No. 21778013)、河北省自然科学基金(No. B2018201234)和河北省高等学校科学技术研究(No. QN2017015)资助项目.
} 
CO 的传统检测方法包括电化学法、气相色谱法和 比色法, 这些都不适合活细胞和活体中实时、动态观测 CO. 荧光方法因其具有选择性好、灵敏度高, 尤其是对 生物样本无侵入性损伤等特点, 被广泛应用于动植物细 胞、组织及活体内内源性活性分子的实时动态检 测 ${ }^{[15 ~ 19]}$. CO 的化学性质相对稳定, 在生理条件下, 能直 接参与的化学反应较少. 与检测其它内源性信号分子的 荧光探针相比, CO 荧光探针发展相对缓慢, 因此, 设计 选择性好、灵敏度高、可用于动态监测活细胞和组织内 $\mathrm{CO}$ 水平变化的荧光探针, 已成为生物医学发展中具有 挑战性的前沿课题之一. 根据近十年来 $\mathrm{CO}$ 荧光探针在 设计、作用机制和生物应用等方面的进展, 本文将根据 荧光探针与 $\mathrm{CO}$ 之间的化学反应类型, 将近十年来所开 发的 $\mathrm{CO}$ 荧光探针按照反应类型进行分类和总结, 综述 $\mathrm{CO}$ 苂光探针的研究进展, 着重概述各相关荧光探针的 设计理念、检测机理及其生物应用, 同时探讨了探针的 结构和性能之间的关系.

\section{1 设计 CO 探针的主要化学反应}

\section{1 环钯金属配合物的羰基化反应或质子化水解反应}

钯是一种过渡金属, 具有配位能力, 含钯金属配合 物已被广泛用于催化、有机合成和药物化学等领域. 金 属钯可以通过重原子效应淬灭荧光团的荧光, 因此, 可 利用金属钯与具有合适配位结构单元的苂光团发生配 位, 制备环钯金属配合物苂光探针(图 1). CO 具有还原 性, 可还原 $\mathrm{Pd}^{2+}$ 为 $\mathrm{Pd}^{0}$, 同时发生羰基化反应或者质子 化水解反应, 释放 $\mathrm{Pd}^{0}$, 此时, 配位键被破坏, 重原子效 应消失, 荧光团荧光恢复. 该类探针设计原理明确, 可 设计荧光打开型探针.

\section{2 零价钯介导的 Tsuji-Trost 反应}

Tsuji-Trost 反应是指烯丙醇及其衍生物烯丙基卤化 物、酯、碳酸盐, 磷酸盐等作为反底物, 在零价钯的催 化作用下，与多种亲核试剂发生取代反应. 含有羟基/胺
基的荧光团，可以与 3 -溴丙烯/氯甲酸烯丙酯反应，形成 烯丙基醚/碳酸酯/氨基甲酸酯等探针结构. CO 具有还原 性, 将外加的 $\mathrm{Pd}^{2+}$ 还原为 $\mathrm{Pd}^{0}, \mathrm{Pd}^{0}$ 进一步介导 Tsuji-Trost 反应的发生, 释放出荧光团. 这类探针通过调控荧光团 上羟基/胺基的给电子能力, 将引起探针分子内部电荷 转移(ICT)机理诱导的苂光最大发射波长蓝移/红移(图 2). 依托该机理可设计用于细胞内 $\mathrm{CO}$ 检测的比率型荧 光探针. 该类探针设计原理明确, 所得的苂光现象为增 强或红移.

\section{3 芳香硝基还原为氨基反应}

典型的 ICT 苂光分子探针是在苂光团上分别连接 强给电子基团和吸电子基团，构成一个强推拉电子体 系，其中荧光团与给电子基团和吸电子基团共轭相连. $\mathrm{CO}$ 可将吸电子基团硝基还原成给电子基团氨基，可以 影响探针分子的电子密度布局，从而达到设计苂光探针 的目的(图 3). 该类探针设计原理明确, 所得的苂光现象 为增强。

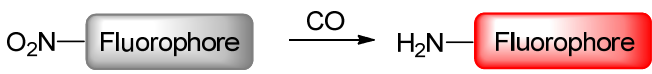

图 3 利用 $\mathrm{CO}$ 还原反应的苂光探针

Figure 3 Fluorescent probes based on the reduction of $\mathrm{CO}$

\section{2 基于金属钯介导的羰基化反应或质子化水解 反应的 $\mathrm{CO}$ 荧光探针}

环钯金属配合物是最早发现用于检测 $\mathrm{CO}$ 的苂光探 针之一, 其主要利用金属钯的重原子效应淬灭探针的荧 光，钯介导探针发生羰基化反应或质子化水解反应，释 放金属钯，实现对 $\mathrm{CO}$ 的打开型荧光响应.

2012 年, Chang 等 ${ }^{[20]}$ 报道了首例 CO 环钯金属配合 物探针 1 (图 4). 该探针以氟嗍吡咯为荧光团, $N, N$-二甲 基苄胺为钯配位基团. 由于金属钯的重原子淬灭效应, 探针 1 荧光较弱; 与 $\mathrm{CO}$ 发生羰基化反应后, 释放出 $\mathrm{Pd}^{0}$, 重原子淬灭消失，探针荧光恢复. 探针对 $\mathrm{CO}$ 具有高选
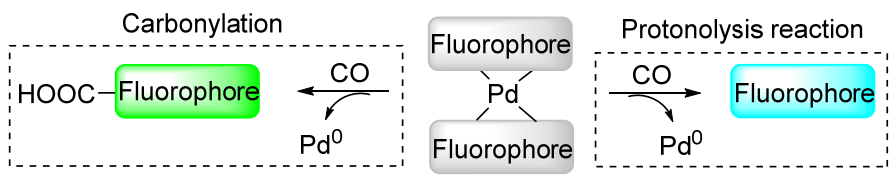

图 1 利用金属钯介导的羰基化反应(左)和质子化水解反应(右)的荧光探针

Figure 1 Fluorescent probes based on the palladium-mediated carbonylation (left) and a protonolysis reaction (right)
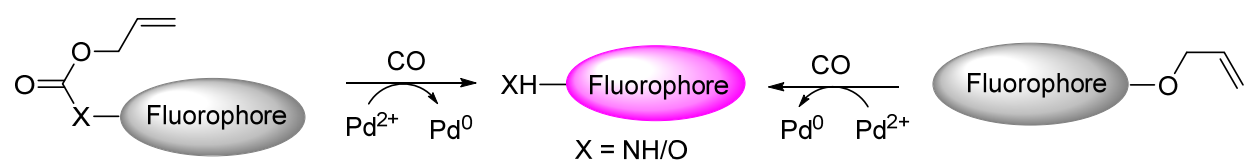

图 2 利用零价钯的 Tsuji-Trost 反应的苂光探针

Figure 2 Fluorescent probes based on palladium(0)-mediated Tsuji-Trost reaction 


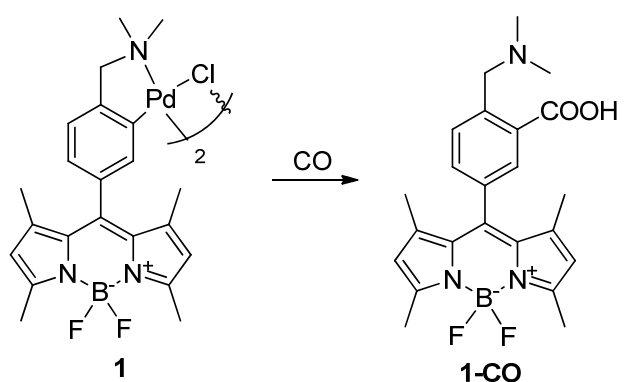

图 4 探针 1 与 $\mathrm{CO}$ 的识别示意图

Figure 4 Schematic illustration of reaction of 1 with CO

择性, 不受其它活性氧物种、活性氮物种、活性硫物种 的干扰. CORM-3 是一种方便、安全的 CO 供体, 在探针 $1\left(1 \mu \mathrm{mol} \cdot \mathrm{L}^{-1}\right)$ 的磷酸盐缓冲液( $\mathrm{PBS}, \mathrm{pH}$ 7.4)中, 加入 50 equiv. 的 CORM-3 分子(水溶性 CO 供体), 随着反应的进 行, 探针溶液苂光逐渐增强, 反应 $60 \mathrm{~min}$ 后, 荧光增强 约 10 倍. 浓度滴定结果表明, 探针的最低检测限为 1 $\mu \mathrm{mol} \cdot \mathrm{L}^{-1}$. 同时, 该探针被用于 HEK293 细胞内 $\mathrm{CO}$ 的 检测.

2014 年, 受 Chang 等工作的启发, 林伟英课题 组 ${ }^{[21]}$ 报道了第一个双光子 $\mathrm{CO}$ 环钯金属配合物荧光探针 2 (图 5). 该探针使用咔唑-香豆素双光子染料平台, 在 $740 \mathrm{~nm}$ 激发下, 产物 2-CO 的双光子吸收截面高达 50.1 GM. 探针 2 对 $\mathrm{CO}$ 具有较高的选择性, 在探针 2 (1 $\mu \mathrm{mol} \cdot \mathrm{L}^{-1}$ ) 的 PBS 溶液(含 $10 \% \mathrm{DMSO}$ )中, 加入不同浓度 的 CORM-2 $\left(0 \sim 120 \mu \mathrm{mol} \cdot \mathrm{L}^{-1}\right)$, 苂光增强 11 倍, 检测极 限为 $0.653 \mu \mathrm{mol} \cdot \mathrm{L}^{-1}$. 利用双光子共聚焦显微成像, 可 以清晰的观察到小鼠肝组织切片 $180 \mu \mathrm{m}$ 深度的 CO. 与 探针 1 不同，该探针与 $\mathrm{CO}$ 发生质子化水解反应.

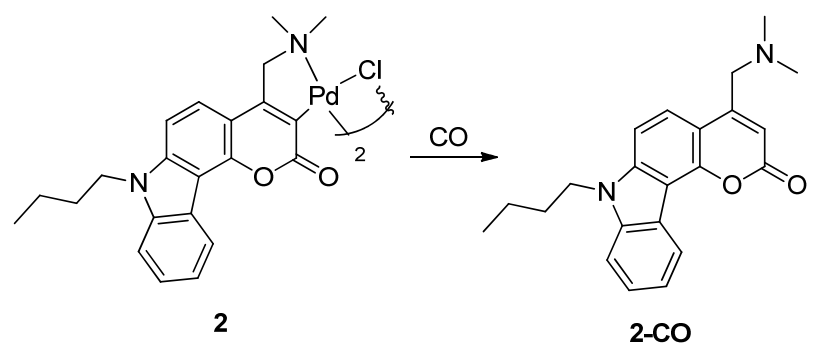

图 5 探针 2 与 $\mathrm{CO}$ 的识别示意图

Figure 5 Schematic illustration of reaction of $\mathbf{2}$ with $\mathrm{CO}$

2016 年, 唐波课题组 ${ }^{[2]}$ 同样以氟嗍吡咯为荧光团, 偶氮苯为钯配位基团, 设计合成了两个环钯金属配合物 $\mathrm{CO}$ 苂光探针 3 和 4 (图 6). 探针与 $\mathrm{CO}$ 发生质子化水解 反应, 释放荧光团的荧光. 在探针 $\left(5 \mu \mathrm{mol} \cdot \mathrm{L}^{-1}\right)$ 的 $\mathrm{PBS}$ 溶液(含 30\% DMSO)中, 加入 20 equiv.的 CORM-2 分子 (CO 供体分子), 反应 $20 \mathrm{~min}$ 后, 探针 3 和 4 苂光增强分 别为 2.5 倍和 10 倍. 在 $0 \sim 80 \mu \mathrm{mol} \cdot \mathrm{L}^{-1}$ 浓度的 CORM-2
范围内, 探针 4 线性关系良好, 检测极限为 $0.72 \mu \mathrm{mol} \cdot$ $\mathrm{L}^{-1}$. 同时, 该探针被首次用于检测乏氧和缺血再灌注 过程中, HepG2 细胞内源 $\mathrm{CO}$ 的诱导生成成像.
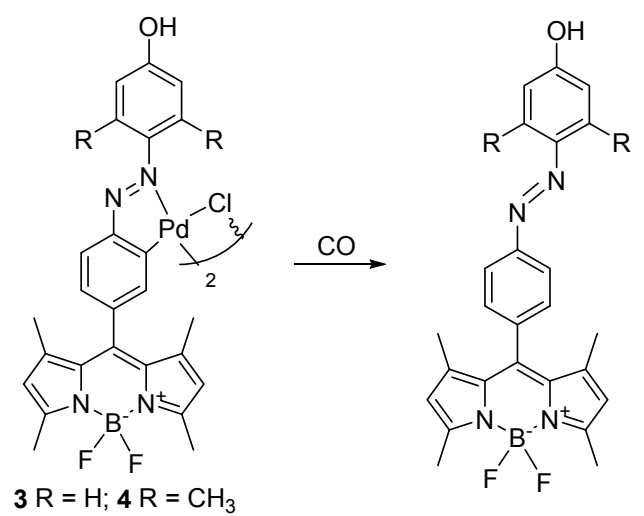

图 6 探针 3 4 与 $\mathrm{CO}$ 的识别示意图

Figure 6 Schematic illustration of reaction of $3 \sim \mathbf{4}$ with $\mathrm{CO}$

2017 年，林伟英课题组 ${ }^{[23,24]}$ 报道了首例双光子激发 近红外发射的环钯金属配合物 CO 苂光探针 5 (图 7). 该 探针以商品化尼罗红为苂光团, 具有合成简便、双光子 激发、长波长发射等优点. 探针本身存在刚性共轭体系, 具有较大的荧光增强倍数和较低的检测极限. 在探针 $(2$ $\mu \mathrm{mol} \cdot \mathrm{L}^{-1}$ ) 的 PBS 溶液(含 $5 \% \mathrm{DMSO}$ )中, 加入 100 equiv. 的 CORM-2, 反应 $60 \mathrm{~min}$ 后，荧光增强高达 60 倍，检测 极限低至 $50 \mathrm{nmol} \cdot \mathrm{L}^{-1}$, 是目前报道的选择性和灵敏性 最好的环钯金属配合物 $\mathrm{CO}$ 探针. 同时，探针双光子激 发波长为 $760 \mathrm{~nm}$, 最大发射波长为 $660 \mathrm{~nm}$, 被用于斑马 鱼胚胎和小鼠组织内源 $\mathrm{CO}$ 的示踪. 小鼠体内成像结果 表明，探针 5 在活体内响应快速，具有较高的分辨率.

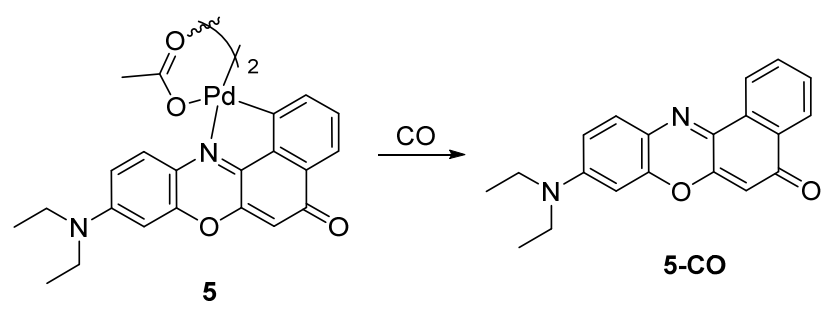

图 7 探针 $\mathbf{5}$ 与 $\mathrm{CO}$ 的识别示意图

Figure 7 Schematic illustration of reaction of 5 with $\mathrm{CO}$

2018 年, 张晓兵课题组 ${ }^{[25]}$ 报道了首个靶向于细胞 膜的近红外环钯金属配合物 CO 苂光探针 6 (图 8). 该探 针同样以尼罗红为荧光团，探针 $\left(5 \mu \mathrm{mol} \cdot \mathrm{L}^{-1}\right)$ 与 100 equiv. 的 CORM-2 反应 $30 \mathrm{~min}$ 后, 苂光增强 25 倍, 检测 极限为 $0.23 \mu \mathrm{mol} \cdot \mathrm{L}^{-1}$. 长疏水烷基链作为膜定位基团, 使探针 6 可以快速针定 $(<1 \mathrm{~min})$ 并长时间滞留 $(>60$ $\min$ )于细胞膜，成功用于检测脂多糖和血红素诱导内源 $\mathrm{CO}$ 生成. 利用探针 6 同时证明, 药物引发肝损伤过程主 要诱导肝脏细胞产生 $\mathrm{CO}$, 并起到保护肝脏细胞的作用. 
同年，王素华课题组 ${ }^{[26]}$ 也报道了一例环钯金属配合物 $\mathrm{CO}$ 荧光探针.

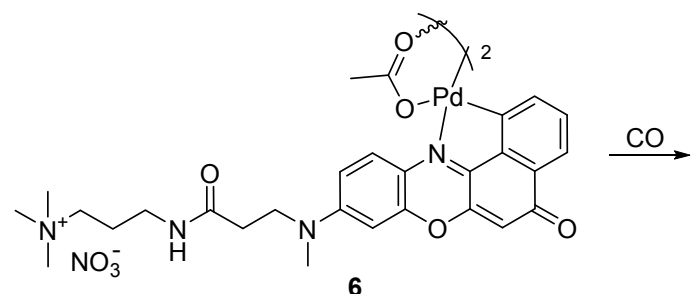<smiles>CN(CCC(=O)NCCC[N+](C)(C)C)c1ccc2nc3c4ccccc4c(=O)cc-3oc2c1</smiles>

图 8 探针 6 与 $\mathrm{CO}$ 的识别示意图

Figure 8 Schematic illustration of reaction of 6 with $\mathrm{CO}$

\section{3 基于零价钯介导 Tsuji-Trost 反应的二组分 CO 荧光探针}

基于零价钯介导 Tsuji-Trost 反应的 CO 苂光探针是 近年来发展快速的一类 $\mathrm{CO}$ 苂光探针, 主要有烯丙基醚/ 烯丙基碳酸酯/烯丙基氨基甲酸酯三种，具有构建灵活、 选择性好、灵敏度高、响应速度快等优点. 此类探针利 用 $\mathrm{CO}$ 还原 $\mathrm{Pd}^{2+}$ 为 $\mathrm{Pd}^{0}, \mathrm{Pd}^{0}$ 进一步介导 $\mathrm{Tsuji}$-Trost 反应 的发生, 产生苂光信号的改变. 此类探针使用时需要外 加钯盐，属于二组分 CO 苂光探针. 按照荧光团的不同, 大致分为: 香豆素类、菜酰亚胺类、氧杂葱类、近红外 区染料类等.

\section{1 以香豆素为苂光团的二组分 $C O$ 荧光探针}

香豆素类苂光团具有合成简便、苂光量子产率高和 光稳定好等优点. 2015 年, Dhara 等 ${ }^{[27]}$ 设计合成了第一个 基于零价钯介导 Tsuji-Trost 反应的 CO 苂光探针 7(图 9). 香豆素 7 位羟基形成氨基甲酸酯后，降低了羟基给电子 能力, 从而抑制了香豆素分子内电荷转移能力, 淬灭其 苂光. $\mathrm{CO}$ 还原 $\mathrm{PdCl}_{2}$ 原位释放 $\mathrm{Pd}^{0}$, 进而发生 Tsuji-Trost 反应，脱除烯丙基氨基碳酸酯. 裸露的仲胺诱导发生分 子内环化-消除反应，释放出 7-羟基香豆素的苂光. 由 于多步反应, 探针 7 与 $\mathrm{CO}$ 的响应速率未见提高, 但是 其检测灵敏性明显高于环钯金属配合物类探针，在探针 $\left(10 \mu \mathrm{mol} \cdot \mathrm{L}^{-1}\right)$ 的 $\mathrm{PBS}$ 溶液(含 $0.4 \% \mathrm{DMSO}$ )中, 加入 5 equiv. 的 CORM-3, 反应 $30 \mathrm{~min}$ 后, 苂光增强高达 130 倍，检测极限低至 $8.49 \mathrm{nmol} \cdot \mathrm{L}^{-1}$.

2016 年, 冯国强课题组 ${ }^{[28]}$ 报道了烯丙基碳酸酯 CO 苂光探针 8(图 10). 该探针以 3-苯并噻唑-7-羟基香豆素 为苂光团，发射波长红移至 $495 \mathrm{~nm}$. 探针对 CO 的响应 速度快、选择性好、灵敏度高，检测极限为 $25 \mathrm{nmol}$ $\mathrm{L}^{-1}$. 探针与 $\mathrm{CO}$ 反应后, 溶液颜色由无色变为黄绿色, 裸眼可观测明显的颜色变化，可用于比色苂光双通道检 测 $\mathrm{CO}$.

\section{2 以萗酰亚胺为荧光团的二组分 $\mathrm{CO}$ 荧光探针}

打开型苂光探针产生单波长荧光信号变化，测试结 果容易受环境因素干扰. 比例计量型荧光探针可以产生 双波长荧光信号变化，可利用双波长比例内矫正的功 能，在一定程度上修正环境对测试结果的影响。

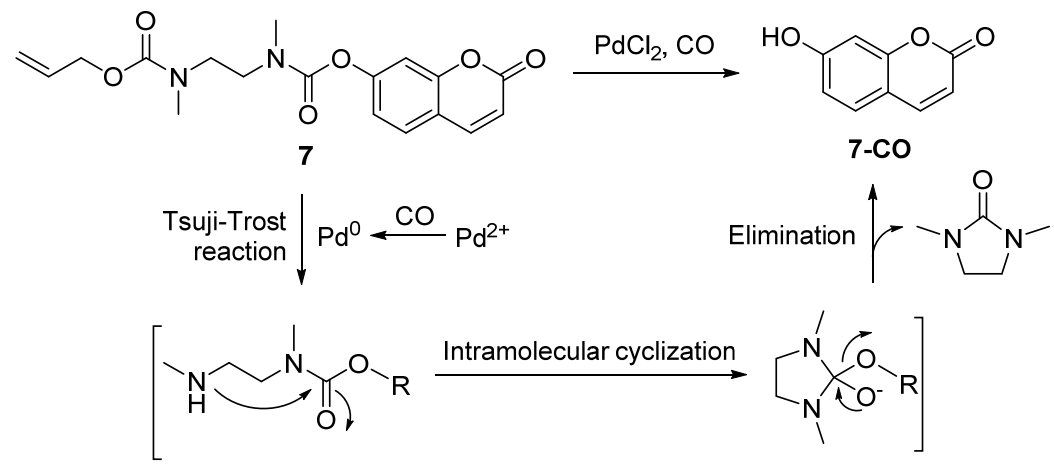

图 9 探针 7 与 $\mathrm{CO}$ 的识别示意图

Figure 9 Schematic illustration of reaction of 7 with $\mathrm{CO}$

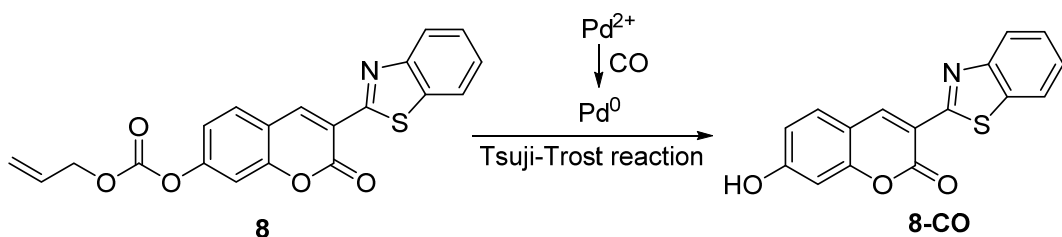

图 10 探针 8 与 $\mathrm{CO}$ 的识别示意图

Figure 10 Schematic illustration of reaction of $\mathbf{8}$ with $\mathrm{CO}$ 
菜酰亚胺是一类经典的具有强推拉电子体系的苂 光染料, 具有合成简便、荧光量子产率高、斯托克斯位 移大等优点, 常被用于设计比例计量型荧光探针. 2017 年, 冯国强课题组 ${ }^{[2]}$ 报道了第一例比例计量型 $\mathrm{CO}$ 荧光 探针 9(图 11). 该探针以 4-胺基荎酰亚胺为荧光团, 烯 丙基氨基甲酸酯为识别基团. 探针结构中 4 位胺基形成 氨基甲酸酯, 降低了氨基给电子能力, 从而降低了芸酰 亚胺分子内电荷转移能力, 使探针发射青色荧光; 在

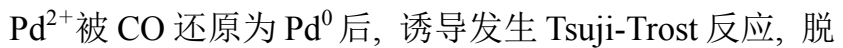
除烯丙基氨基碳酸酯, 释放的胺基增加了分子内电荷转 移能力, 使探针发射出黄绿色荧光. 探针对 $\mathrm{CO}$ 具有高 选择性和高灵敏性, 在探针 $\left(10 \mu \mathrm{mol} \cdot \mathrm{L}^{-1}\right)$ 的 PBS 溶液 (含 $30 \%$ DMSO) 中, 加入 10 equiv.的 CORM-3, 反应 20 $\mathrm{min}$ 后, 比率苂光增强 16.7 倍, 检测极限为 $58 \mathrm{nmol}$ $\mathrm{L}^{-1}$, 被用于溶液中 $\mathrm{CO}$ 检测、细胞内源 $\mathrm{CO}$ 的苂光成像.
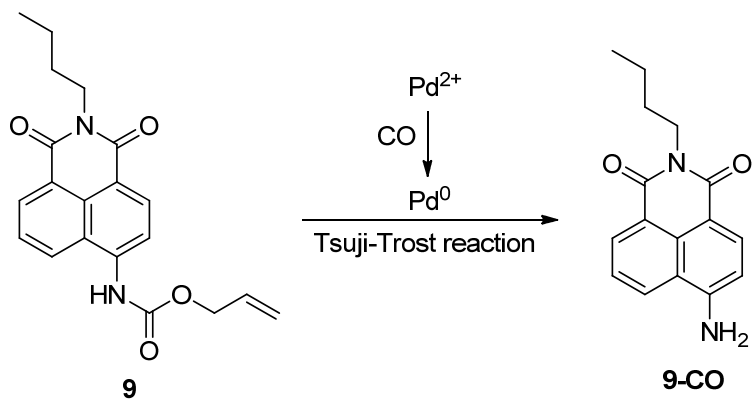

图 11 探针 9 与 $\mathrm{CO}$ 的识别示意图

Figure 11 Schematic illustration of reaction of 9 with $\mathrm{CO}$

2018 年, 朱宝存课题组 ${ }^{[30]}$ 报道了第二例比例计量 型 $\mathrm{CO}$ 菼光探针 10(图 12). 该探针以 4-羟基䒬酰亚胺为 荧光团, 烯丙基醚为识别基团, 探针对 CO 选择性高于 其它活性物种，具有较高选择性，检测极限为 17.9 $\mathrm{nmol} \cdot \mathrm{L}^{-1}$ ，荧光双发射波长斯托克斯位移高达 $90 \mathrm{~nm}$.
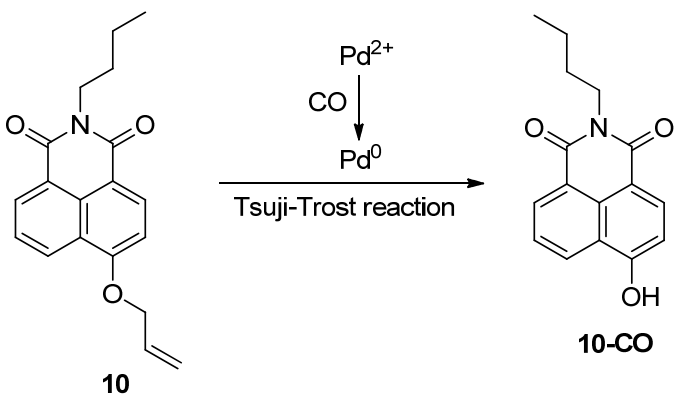

图 12 探针 10 与 $\mathrm{CO}$ 的识别示意图

Figure 12 Schematic illustration of reaction of $\mathbf{1 0}$ with $\mathrm{CO}$

\section{3 以氧杂葱为荧光团的二组分 $\mathrm{CO}$ 荧光探针}

氧杂葱是一类光学性能优异的染料分子, 包括苂光 素、罗丹明、Rhodol 及其类似物, 普遍具有较大的摩尔
消光系数和较高的荧光量子产率, 被广泛应用于分子识 别、生物化学和医学研究等领域.

当前, 利用氧杂葱类染料发展的二组分 CO 苂光探 针主要是利用羟基的保护和脱保护策略. 两个羟基都被 保护的荧光素形成非共轭结构的无色无荧光闭环内酯 结构; 单羟基保护的 Rhodol 的苂光也显著下降，当分析 物促进羟基脱保护后，重新释放染料的苂光.

2016 年, 冯国强课题组 ${ }^{[31]}$ 报道了双烯丙基碳酸酯 CO 苂光探针 11(图 13). 该探针以苂光素为苂光团, 通 过与 $\mathrm{CO}$ 反应破坏螺内酯结构, 释放荧光素的荧光. 该 探针对 CO 具有高选择性, 不受其它活性氧、活性氮、 活性硫物种的干扰. 在探针 $\left(5 \mu \mathrm{mol} \cdot \mathrm{L}^{-1}\right.$ ) 的 PBS 溶液(含 $0.5 \% \mathrm{DMSO})$ 中, 加入 10 equiv.的 CORM-3，反应 $15 \mathrm{~min}$ 后，苂光增强约 100 倍，检测极限为 $37 \mathrm{nmol} \cdot \mathrm{L}^{-1}$. 探针 用于检测血红素诱导 A549 细胞内源 CO 的生成. 随后, 该课题组 ${ }^{[32]}$ 又报道了双烯丙基醚 $\mathrm{CO}$ 荧光探针 12 (图 13). 该探针利用更稳定的烯丙基醚代替了烯丙基碳酸 酯作为反应位点，克服了烯丙基碳酸酯探针 11 稳定性 差的问题. 该探针结构简单、化学/光稳定良好、对 $\mathrm{CO}$ 具有较高的选择性和灵敏性, 检测极限为 $29 \mathrm{nmol} \cdot \mathrm{L}^{-1}$.

2016 年, 张雷课题组 ${ }^{[33]}$ 首次报道了双烯丙基碳酸 酯近红外荧光 $\mathrm{CO}$ 探针 13 (图 14). 该探针以荎基荧光素 为苂光团, 烯丙基碳酸酯为反应基团, 对 $\mathrm{CO}$ 选择性和 灵敏度高，在探针 $\left(10 \mu \mathrm{mol} \cdot \mathrm{L}^{-1}\right)$ 的 PBS 溶液(含 $40 \%$ DMSO)中, 加入 10 equiv.的 CORM-3, 反应 $45 \mathrm{~min}$ 后, 苂光增强 35 倍, 检测极限 $127 \mathrm{nmol} \cdot \mathrm{L}^{-1}$, 可实现 $\mathrm{CO}$ 比 色荧光双通道检测. 2019 年, 朱宝存课题组 ${ }^{[34]}$ 报道了烯 丙基碳酸酯 CO 苂光探针 14(图 15). 该探针以近红外氧 杂葱染料 Seminaphthorhodafluor 为荧光团, 对 $\mathrm{CO}$ 选择 性高于其它活性氧、活性氮、活性硫等物种. 探针检测 极限为 $38.9 \mathrm{nmol} \cdot \mathrm{L}^{-1}$, 能够用于 $\mathrm{HeLa}$ 细胞线粒体内 $\mathrm{CO}$ 的荧光成像.

\section{4 以近红外区荧光类染料的二组分 $C O$ 荧光探针}

近红外荧光探针的激发发射波长一般位于 650 $900 \mathrm{~nm}$, 该波长范围内生物背景干扰低, 可获得较高的 成像信噪比, 同时, 该波长区域内光子的辐射能较低, 可减小光对生物样本的损伤. 另外, 近红外光组织穿透 能力强, 可以实现活体动物体内荧光成像. 常用的近红 外苂光团是菁染料、长沙系列染料、湖大系列染料等, 此 类荧光团具有较大的摩尔消光系数、易于合成和纯化等 优点，已经被广泛应用于许多领域.

2018 年, 冯国强课题组 ${ }^{[35]}$ 设计合成了烯丙基碳酸 酯近红外苂光 CO 探针 $\mathbf{1 5}$ (图 16). 该探针以湖大染料为 荧光团, 对 $\mathrm{CO}$ 具有较好的选择性和较高的灵敏度, 检 测极限低至 $3.2 \mathrm{nmol} \cdot \mathrm{L}^{-1}$. 探针水溶性好, 与 $\mathrm{CO}$ 几分钟 


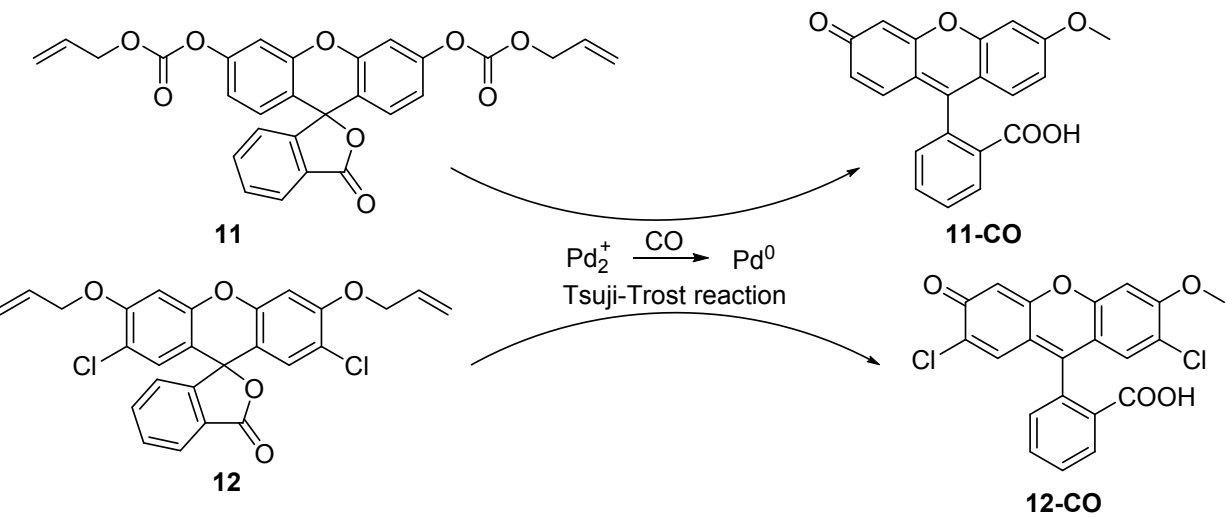

图 13 探针 11 12 与 $\mathrm{CO}$ 的识别示意图

Figure 13 Schematic illustration of reaction of $\mathbf{1 1} \sim \mathbf{1 2}$ with $\mathrm{CO}$
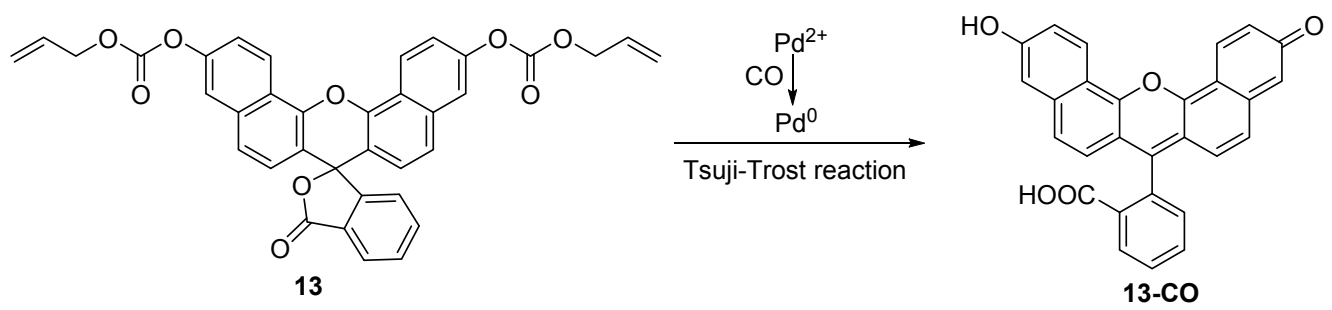

图 14 探针 13 与 $\mathrm{CO}$ 的识别示意图

Figure 14 Schematic illustration of reaction of 13 with $\mathrm{CO}$

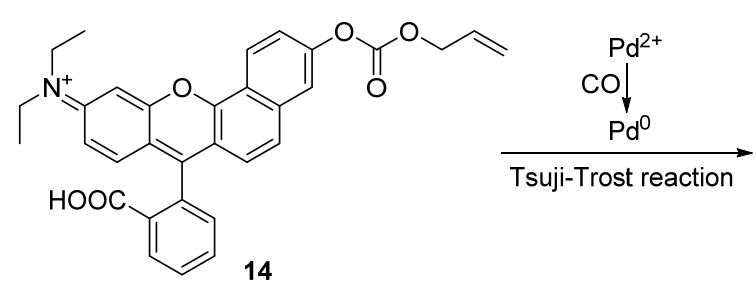<smiles></smiles>

图 15 探针 14 与 $\mathrm{CO}$ 的识别示意图

Figure 15 Schematic illustration of reaction of 14 with CO

内即可反应完全, 并表现出明显的颜色和荧光变化. 该 探针被用于 $\mathrm{HeLa}$ 细胞和小鼠体内 $\mathrm{CO}$ 的苂光成像. 同 年, 李春艳课题组 ${ }^{[36]}$ 同样报道了一例以湖大染料类似 物为荧光团的烯丙基碳酸酯近红外苂光 $\mathrm{CO}$ 探针 16 (图 16), 该探针实现了细胞线粒体和小鼠体内 $\mathrm{CO}$ 的荧光成 像.

2019 年, 冯国强课题组 ${ }^{[37,38]}$ 先后报道了两个大斯托 克斯位移的烯丙基碳酸酯近红外苂光 CO 探针(图 16). 探针 17 和 18 对 $\mathrm{CO}$ 针都具有高选择性和灵敏度, 检测 极性低至纳摩尔级, 它们的斯托克斯位移均高于 200 $\mathrm{nm}$, 实现了细胞、斑马鱼和小鼠体内 $\mathrm{CO}$ 的荧光成像.

\section{5 以其他染料为苂光团的二组分 $C O$ 荧光探针}

2016 年, 张雷课题组 ${ }^{[39]}$ 报道了基于分子内电荷转 移(ICT)机理的比色和苂光 CO 探针 19(图 17). 该探针以 硝基苯并呋咱(NBD)为荧光团，烯丙基氨基甲酸酯为反

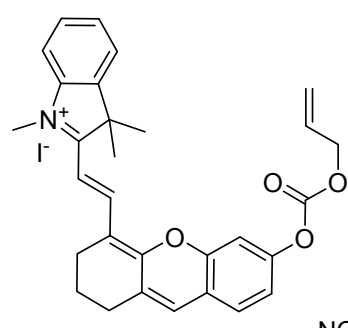

15<smiles>C=CCOC(=O)Oc1ccc2c(c1)OC1=C(/C=C/C3=[N+](CC)c4ccc5ccccc5c4C3(C)C)CCCC1=C2</smiles>

16<smiles>C=CCOC(=O)Oc1ccc(/C=C/C2=CCC(C)(C)CC2=[In])cc1</smiles>

图 16 探针 15 18 的结构

Figure 16 Structures of probes $15 \sim 18$ 
应基团, 对 $\mathrm{CO}$ 具有优异的选择性, 超过其他的活性氧、 活性硫和活性氮物种. 在探针 $\left(10 \mu \mathrm{mol} \bullet \mathrm{L}^{-1}\right)$ 的 PBS 溶液 中, 加入 5 equiv. 的 CORM-3, 反应 $30 \mathrm{~min}$ 后, 苂光增强 75 倍, 检测极限 $26.3 \mathrm{nmol} \cdot \mathrm{L}^{-1}$, 可实现 CO 比色苂光双 通道检测. 该探针被用于比色检测空气中 $\mathrm{CO}$ 气体的存 在.<smiles>C=CCOC(=O)N(CCC)c1ccc([N+](=O)[O-])c2nonc12</smiles>
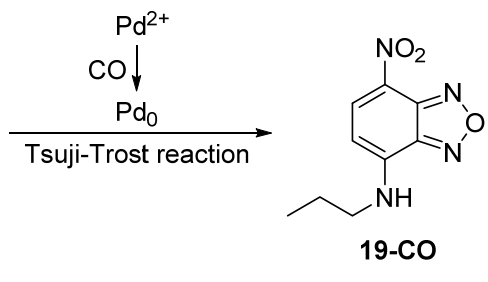

图 17 探针 19 与 $\mathrm{CO}$ 的识别示意图

Figure 17 Schematic illustration of reaction of 19 with CO

\section{4 基于芳香硝基还原为氨基反应的 $\mathrm{CO}$ 荧光探 针}

$\mathrm{CO}$ 具有还原性，一些芳香硝基化合物理论上能够 被其还原, 因此, 寻找合适结构的芳香硝基化合物有望 实现 $\mathrm{CO}$ 的选择性检测.

2018 年, Dhara 等 ${ }^{[40,41]}$ 报道了两例基于一氧化碳还 原反应的 CO 荧光探针 20 和 21 (图 18). 两个探针结构中 萗酰亚胺 3 位硝基被还原为胺基后, 恢复了苂光团的苂 光. 它们对 $\mathrm{CO}$ 具有较高的选择性和灵敏性, 在探针(10 $\mu \mathrm{mol} \cdot \mathrm{L}^{-1}$ ) 的 $\mathrm{PBS}$ 溶液(含 $1 \sim 2 \% \mathrm{DMSO}$ )中, 加入 10 equiv. 的 CORM-3, 反应 $45 \mathrm{~min}$ 后, 荧光分别增强 33 倍 和 75 倍, 检测极限可达纳摩尔级别. 探针 21 能够定位 于 MCF-7 细胞溶酶体, 用于溶酶体内 CO 的苂光成像.

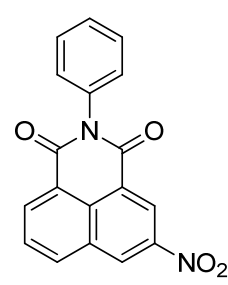

20

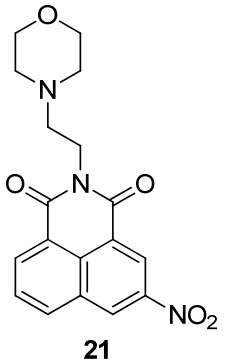

21
图 18 探针 $20 \sim 21$ 的结构

Figure 18 Structures of probes $\mathbf{2 0} \sim \mathbf{2 1}$

2019 年, 朱宝存课题组 ${ }^{[42]}$ 设计合成了首个基于一 氧化碳还原反应的近红外 CO 荧光探针 22 (图 19). 该探 针对 CO 的选择性远超过其他的活性氧、活性硫和活性 氮物种. 在探针 $\left(10 \mu \mathrm{mol} \cdot \mathrm{L}^{-1}\right)$ 的 $\mathrm{PBS}$ 溶液(含 $30 \%$ DMSO)中, 加入 10 equiv. 的 CORM-2, 反应 $10 \mathrm{~min}$ 后, 苂光即能增强 $80 \%$, 检测极限低至 $6.1 \mathrm{nmol} \cdot \mathrm{L}^{-1}$, 结合 共聚焦荧光成像技术，首次证实 RAW 264.7 巨噬细胞中
瞬时葡萄糖去氧(TGD)引起血红素氧合酶-1 (HO-1)上调 和高糖抑制斑马鱼 HO-1 下调的现象.

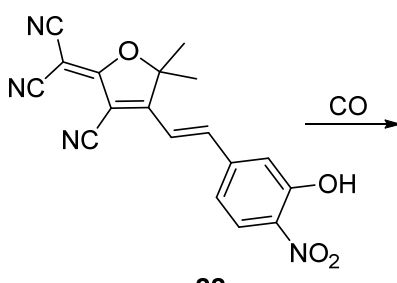

22

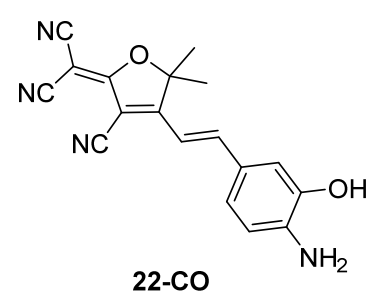

22-CO
图 19 探针 22 与 $\mathrm{CO}$ 的识别示意图

Figure 19 Schematic illustration of reaction of 22 with CO

同年，冯国强课题组 ${ }^{[43]}$ 发现一例基于激发态分子 内质子转移(ESIPT)机制的 CO 荧光探针 23(图 20). 该探 针以 2-硝基邻苯二甲酰亚胺为菼光基团，硝基被 CO 还 原为胺基后，探针发生 ESIPT，产生绿色荧光. 该探针 用于快速、高选择性、灵敏的检测在水溶液、活细胞和 动物中的 CORM-3, 为研究 CORM-3 在生物系统中的应 用提供了有用的工具.<smiles>O=C(O)CN1C(=O)c2cccc([N+](=O)[O-])c2C1=O</smiles>

23

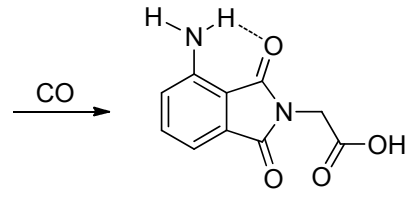

23-CO
图 20 探针 23 与 $\mathrm{CO}$ 的识别示意图

Figure 20 Schematic illustration of reaction of $\mathbf{2 3}$ with CO

\section{5 结论与展望}

近年来 $\mathrm{CO}$ 苂光分子探针的研究已经取得了一定进 展, 见表 1. 由于 $\mathrm{CO}$ 的化学性质相对稳定, 在生理条件 下能直接参与的化学反应较少, 因此, 与检测其它内源 性活性分子的苂光探针相比, CO 苂光探针的研究尚未 成熟, 有广阔的发展空间. 近年来 $\mathrm{CO}$ 荧光分子探针的 研究已经取得了一定进展，一些探针分子已用于细胞和 生物体内 $\mathrm{CO}$ 浓度的检测, 用于研究特定环境(如乏氧、 缺血再灌注损伤等)下内源性 $\mathrm{CO}$ 的生成与代谢关系, 但 适用于研究活体内 $\mathrm{CO}$ 水平与相关疾病的关系的新型长 波长/双光子激发近红外发射性质的荧光分子探针还有 待发展. 另外, 已报道的 CO 苂光探针多为打开型探针, 测试结果易受到探针浓度、激发光源效率和外界环境等 因素的影响，发展比例计量型探针，利用其双波长比例 内校正功能, 有望实现 $\mathrm{CO}$ 精确检测. 最后, 基于零价 钯介导 Tsuji-Trost 反应的 CO 苂光探针是二组分探针, 虽然已经用于 $\mathrm{CO}$ 的定量检测，但实际应用中仍存在一 些不足, 我们认为发展基于 Tsuji-Trost 反应的 CO 单一 组分荧光探针也是 $\mathrm{CO}$ 发展的一个方向. 
表 $1 \mathrm{CO}$ 荧光探针的总结

Table 1 Summary of CO fluorescent probes

\begin{tabular}{|c|c|c|c|c|c|c|c|c|}
\hline 探针 & 探针类型 & 荧光团 & $\begin{array}{l}\lambda_{\mathrm{ex}} / \lambda_{\mathrm{em}} \\
(\mathrm{nm})\end{array}$ & 测试溶液 & 检测极限 & 荧光增强倍数 & $\begin{array}{c}\text { 响应时间/ } \\
\text { min }\end{array}$ & $\begin{array}{c}\text { 应用 } \\
\text { (细胞/活体) }\end{array}$ \\
\hline $1^{[20]}$ & \multirow{6}{*}{$\begin{array}{l}\text { 环钯金属配合物 } \\
\text { CO 苂光探针 }\end{array}$} & 氟硼吡咯 & $475 / 503$ & PBS & $1 \mu \mathrm{mol} \cdot \mathrm{L}^{-1}$ & $\begin{array}{l}10 \text { 倍 }\left(1 \mu \mathrm{mol} \cdot \mathrm{L}^{-1} \text {, }\right. \\
50 \text { equiv. })\end{array}$ & 60 & 细胞 \\
\hline $2^{[21]}$ & & 咔唑-香豆素 & $740 / 477$ & $10 \%$ DMSO & $0.653 \mu \mathrm{mol} \cdot \mathrm{L}^{-1}$ & $\begin{array}{l}11 \text { 倍 }\left(2 \mu \mathrm{mol} \cdot \mathrm{L}^{-1},\right. \\
60 \text { equiv. })\end{array}$ & 40 & 细胞/组织 \\
\hline $3^{[22]}$ & & 氟硼吡咯 & $498 / 512$ & $30 \%$ DMSO & - & $\begin{array}{l}2.5 \text { 倍 }\left(5 \mu \mathrm{mol} \cdot \mathrm{L}^{-1} \text {, }\right. \\
20 \text { equiv.) }\end{array}$ & 20 & - \\
\hline $4^{[23]}$ & & 氟硼吡咯 & $498 / 512$ & $30 \%$ DMSO & $0.72 \mu \mathrm{mol} \cdot \mathrm{L}^{-1}$ & $\begin{array}{l}10 \text { 倍 }\left(5 \mu \mathrm{mol} \cdot \mathrm{L}^{-1} \text {, }\right. \\
20 \text { equiv. })\end{array}$ & 20 & 细胞 \\
\hline $5^{[24]}$ & & 尼罗红 & $760 / 660$ & $5 \%$ DMSO & $50 \mathrm{nmol} \cdot \mathrm{L}^{-1}$ & $\begin{array}{l}60 \text { 倍 }\left(2 \mu \mathrm{mol} \cdot \mathrm{L}^{-1},\right. \\
100 \text { equiv. })\end{array}$ & 60 & $\begin{array}{l}\text { 细胞/斑马鱼/ } \\
\text { 小鼠 }\end{array}$ \\
\hline $6^{[25]}$ & & 尼罗红 & $543 / 650$ & $5 \%$ DMSO & $0.23 \mu \mathrm{mol} \cdot \mathrm{L}^{-1}$ & $\begin{array}{l}25 \text { 倍 }\left(5 \mu \mathrm{mol} \cdot \mathrm{L}^{-1} \text {, }\right. \\
20 \text { equiv. })\end{array}$ & 30 & 细胞膜/小鼠 \\
\hline $7^{[27]}$ & \multirow{13}{*}{$\begin{array}{l}\text { 二组分 CO 苂光 } \\
\text { 探针(烯丙基醚/ } \\
\text { 烯丙基碳酸酯/ } \\
\text { 烯丙基氨基 } \\
\text { 甲酸酯) }\end{array}$} & 香豆素 & $340 / 460$ & $0.4 \%$ DMSO & $7.77 \mathrm{nmol} \cdot \mathrm{L}^{-1}$ & $\begin{array}{l}130 \text { 倍 }\left(10 \mu \mathrm{mol} \cdot \mathrm{L}^{-1} \text {, }\right. \\
5 \text { equiv. })\end{array}$ & 30 & 细胞 \\
\hline $8^{[28]}$ & & $\begin{array}{l}\text { 苯并噻唑 } \\
\text { 香豆素 }\end{array}$ & $462 / 490$ & $10 \%$ DMSO & $25 \mathrm{nmol} \cdot \mathrm{L}^{-1}$ & $\begin{array}{l}42 \text { 倍 }\left(5 \mu \mathrm{mol} \cdot \mathrm{L}^{-1} \text {, }\right. \\
10 \text { equiv. })\end{array}$ & 15 & 细胞 \\
\hline $9^{[29]}$ & & 萫酰亚胺 & $\begin{array}{l}420 / 472, \\
545\end{array}$ & 30\% DMSO & $58 \mathrm{nmol} \cdot \mathrm{L}^{-1}$ & $\begin{array}{l}16.7 \text { 倍 }\left(10 \mu \mathrm{mol} \cdot \mathrm{L}^{-1} \text {, }\right. \\
10 \text { equiv. })\end{array}$ & 20 & 细胞 \\
\hline $10^{[30]}$ & & 萫酰亚胺 & $\begin{array}{l}430 / 455 \\
545\end{array}$ & PBS & $17.9 \mathrm{nmol} \cdot \mathrm{L}^{-1}$ & - & - & 细胞 \\
\hline $11^{[31]}$ & & 苂光素 & $490 / 520$ & $0.5 \%$ DMSO & $37 \mathrm{nmol} \cdot \mathrm{L}^{-1}$ & $\begin{array}{l}100 \text { 倍 }\left(5 \mu \mathrm{mol} \cdot \mathrm{L}^{-1} \text {, }\right. \\
10 \text { equiv. })\end{array}$ & 15 & 细胞 \\
\hline $12^{[32]}$ & & 荧光素 & $493 / 527$ & $0.5 \%$ DMSO & $29 \mathrm{nmol} \cdot \mathrm{L}^{-1}$ & $\begin{array}{l}100 \text { 倍 }\left(5 \mu \mathrm{mol} \cdot \mathrm{L}^{-1} \text {, }\right. \\
20 \text { equiv. })\end{array}$ & 20 & 细胞 \\
\hline $13^{[33]}$ & & 萘基荧光素 & $620 / 670$ & $40 \%$ DMSO & $0.127 \mathrm{nmol} \cdot \mathrm{L}^{-1}$ & $\begin{array}{l}35 \text { 倍 }\left(1 \mu \mathrm{mol} \cdot \mathrm{L}^{-1} \text {, }\right. \\
10 \text { equiv. })\end{array}$ & 45 & 细胞 \\
\hline $14^{[34]}$ & & $\begin{array}{l}\text { Seminaphtho- } \\
\text { rhodafluor }\end{array}$ & $450 / 630$ & PBS & $38.9 \mathrm{nmol} \cdot \mathrm{L}^{-1}$ & $\begin{array}{l}20 \text { 倍 }\left(5 \mu \mathrm{mol} \cdot \mathrm{L}^{-1} \text {, }\right. \\
10 \text { equiv. })\end{array}$ & 20 & 线粒体 \\
\hline $15^{[35]}$ & & 湖大染料 & $670 / 714$ & $0.5 \%$ DMSO & $3.2 \mathrm{nmol} \cdot \mathrm{L}^{-1}$ & - & - & 细胞/小鼠 \\
\hline $16^{[36]}$ & & 湖大染料 & $690 / 736$ & PBS & $0.17 \mathrm{nmol} \cdot \mathrm{L}^{-1}$ & - & - & 线粒体/小鼠 \\
\hline $17^{[37]}$ & & $\begin{array}{l}\text { 异佛尔酮 } \\
\text { 衍生物 }\end{array}$ & $550 / 671$ & $20 \%$ DMSO & $38 \mathrm{nmol} \cdot \mathrm{L}^{-1}$ & - & - & 细胞/小鼠 \\
\hline $18^{[38]}$ & & $\begin{array}{l}\text { 异佛尔酮- } \\
\text { 香豆素 }\end{array}$ & $510 / 710$ & $5 \%$ DMSO & $33 \mathrm{nmol} \cdot \mathrm{L}^{-1}$ & - & - & 细胞/斑马鱼 \\
\hline $19^{[39]}$ & & $\begin{array}{l}\text { 7-硝基-2,1,3- } \\
\text { 苯并呋咱 }\end{array}$ & $480 / 549$ & PBS & $263 \mathrm{nmol} \cdot \mathrm{L}^{-1}$ & $\begin{array}{l}75 \text { 倍( } 10 \mu \mathrm{mol} \cdot \mathrm{L}^{-1} \text {, } \\
5 \text { equiv. })\end{array}$ & 30 & 细胞 \\
\hline $20^{[40]}$ & \multirow{4}{*}{$\begin{array}{l}\text { 硝基还原型 } \\
\mathrm{CO} \text { 苂光探 } \\
\text { 针 }\end{array}$} & 蒜酰亚胺 & $440 / 522$ & $2 \% \mathrm{DMSO}$ & $123 \mathrm{nmol} \cdot \mathrm{L}^{-1}$ & $\begin{array}{l}33 \text { 倍( } 10 \mu \mathrm{mol} \cdot \mathrm{L}^{-1} \text {, } \\
10 \text { equiv. })\end{array}$ & 45 & 细胞 \\
\hline $21^{[41]}$ & & 芸酰亚胺 & $440 / 528$ & $1 \% \mathrm{DMSO}$ & $0.60 \mathrm{nmol} \cdot \mathrm{L}^{-1}$ & $\begin{array}{l}75 \text { 倍 }\left(10 \mu \mathrm{mol} \cdot \mathrm{L}^{-1} \text {, }\right. \\
10 \text { equiv. })\end{array}$ & 45 & 细胞溶酶体 \\
\hline $22^{[42]}$ & & 多氧基荧光团 & $580 / 665$ & $30 \%$ DMSO & $6.1 \mathrm{nmol} \cdot \mathrm{L}^{-1}$ & $\begin{array}{l}80 \%\left(10 \mu \mathrm{mol} \cdot \mathrm{L}^{-1}\right. \\
10 \text { equiv. })\end{array}$ & 10 & 细胞/斑马鱼 \\
\hline $23^{[43]}$ & & $\begin{array}{l}\text { 2-硝基邻苯二 } \\
\text { 甲酰亚胺 }\end{array}$ & $420 / 503$ & $0.5 \%$ DMSO & $16 \mathrm{nmol} \cdot \mathrm{L}^{-1}$ & $\begin{array}{l}\text { Vast }\left(10 \mu \mathrm{mol} \cdot \mathrm{L}^{-1} \text {, }\right. \\
10 \text { equiv. })\end{array}$ & 5 & $\begin{array}{l}\text { 细胞/斑马鱼/ } \\
\text { 小鼠 }\end{array}$ \\
\hline
\end{tabular}




\section{References}

[1] Motterlini, R.; Otterbein, L. E. Nat. Rev. Drug Discovery 2010, 9, 728.

[2] Sjöstrand, T. Scand. J. Clin. Lab. Invest. 1949, 1, 201.

[3] Muramoto, T.; Tsurui, N.; Terry, M. J.; Yokota, A.; Kohchi, T. Plant Physiol. 2002, 130, 1958.

[4] Muramoto, T.; Kohchi, T.; Yokota, A.; Hwang, I.; Goodman, H. M. Plant Cell 1999, 11, 335.

[5] Xuan, W.; Zhu, F.-Y.; Xu, S.; Huang, B.-K.; Ling, T.-F.; Qi, J.-Y. Plant Physiol. 2008, 148, 881.

[6] Han, Y.; Xuan, W.; Yu, T.; Fang, W. B.; Lou, T. L.; Gao, Y. J. Integr. Plant Biol. 2007, 49, 1703.

[7] Noriega, G. O.; Balestrasse, K. B.; Batlle, A.; Tomaro, M. L. Biochem. Biophys. Res. Commun. 2004, 323, 1003.

[8] Cao, Z.; Huang, B.; Wang, Q.; Xuan, W.; Ling, T.; Zhang, B. Chin. Sci. Bull. 2007, 52, 2365.

[9] Mann, B. E. Top. Organomet. Chem. 2010, 32, 247.

[10] Bilban, M.; Haschemi, A.; Wegiel, B.; Chin, B. Y.; Wagner, O.; Otterbein, L. E. J. Mol. Med. 2008, 86, 267.

[11] Fujimoto, H.; Ohno, M.; Ayabe, S.; Kobayashi, H.; Ishizaka, N.; Kimura, H. Arterioscler., Thromb., Vasc. Biol. 2004, 24, 1848.

[12] Heinemann, S. H.; Hoshi, T.; Westerhausen, M.; Schiller, A. Chem. Commun. 2014, 50, 3644.

[13] Ryter, S. W.; Choi, A. M. K. Transl. Res. 2016, 167, 7.

[14] Wu, L. Y.; Wang, R. Pharmacol. Rev. 2005, 57, 585.

[15] Jiao, C. P.; Liu, Y. Y.; Lu, W. J.; Zhang, P. P.; Wang, Y. F. Chin. J. Org. Chem. 2019, 39, 591 (in Chinese).

(矫春鹏，刘媛媛，路文娟，张平平，王延风，有机化学，2019, 39, 591.)

[16] Yan, P. P.; Wang, T.; Zhang, D.; Ma, X. X. Chin. J. Org. Chem. 2019, 39, 916 (in Chinese). (间沛沛，王婷，张丹，马晓雪，有机化学，2019, 39, 916.)

[17] Yan, P. P.; Wang, T.; Zhang, D.; Ma, X. X. Chin. J. Org. Chem. 2019, 39, 952 (in Chinese). (王瑞祥, 赖晓静, 邱观音生, 刘晋彪, 有机化学, 2019, 39, 952.)

[18] Zhang, S. X.; Niu, Q. M.; Wu, S. Z.; Lv, H. J.; Xing, G. W.; Xing, G. W. Chin. J. Org. Chem. 2019, 39, 940 (in Chinese). (张晟曦, 牛晴旻, 吴松泽, 吕海娟, 邢国文, 有机化学, 2019, 39, 940.)

[19] Yang, Z. Q.; Liu, X. K.; Jiang, L. N.; Wang, M. Chin. J. Org. Chem. 2019, 39, 1483 (in Chinese)

(杨滋琦, 刘兴坤, 姜鲁南, 王美, 有机化学, 2019, 39, 1483.)

[20] Michel, B. W.; Lippert, A. R.; Chang, C. J. J. Am. Chem. Soc. 2012,
$134,15668$.

[21] Zheng, K.; Lin, W.; Tan, L.; Chen, H.; Cui, H. Chem. Sci. 2014, 5, 3439.

[22] Li, Y.; Wang, X.; Yang, J.; Xie, X. L.; Li, M. M.; Niu, J. Y. Anal. Chem. 2016, 88, 11154.

[23] Liu, K. Y.; Kong, X. Q.; Ma, Y. Y.; Lin, W. Y. Angew. Chem. Int. Ed. 2017, 56, 13489.

[24] Liu, K.; Kong, X.; Ma, Y.; Lin, W. Nat. Protoc. 2018, 13, 1020.

[25] Xu, S.; Liu, H.-W.; Yin, X.; Yuan, L.; Huan, S.-Y.; Zhang, X.-B. Chem. Sci. 2018, 10, 320.

[26] Sun, M.; Yu, H.; Zhang, K.; Wang, S.; Hayat, T.; Alsaedi, A. ACS Sens. 2018, 3, 285.

[27] Pal, S.; Mukherjee, M.; Sen, B.; Mandal, S. K.; Lohar, S.; Chattopadhyay, P.; Dhara, K. Chem. Commun. 2015, 51, 4410.

[28] Feng, W. Y.; Liu, D. D.; Zhai, Q. S.; Feng, G. Q. Sens. Actuators, B 2017, 240, 625.

[29] Feng, W. Y.; Hong, J. X.; Feng, G. Q. Sens. Actuators, B 2017, 251, 389 .

[30] Wang, Z.; Geng, Z.; Zhao, Z.; Sheng, W.; Liu, C.; Lv, X. New J. Chem. 2018, 42, 14417.

[31] Feng, W. Y.; Liu, D. D.; Feng, S. M.; Feng, G. Q. Anal. Chem. 2016, 88, 10648 .

[32] Feng, S. M.; Liu, D. D.; Feng, W. Y.; Feng, G. Q. Anal. Chem. 2017, 89, 3754.

[33] Yan, J.-W.; Zhu, J.-Y.; Tan, Q.-F.; Zhou, L.-F.; Yao P.-F.; Lu, Y.-T.; Tan, J.-H.; Zhang, L. RSC Adv. 2016, 6, 65373.

[34] Wang, Z.; Zhao, Z.; Wang, R.; Yuan, R.; Liu, C.; Duan, Q.; Zhu, W.; Li, X.; Zhu, B. Anal. Methods 2019, 11, 288.

[35] Feng, W. Y.; Feng, G. Q. Sens. Actuators B. 2018, 255, 2314.

[36] Li, S.-J.; Zhou, D.-Y.; Li, Y.-F.; Yang, B.; Ou-Yang, J.; Jie, J.; Liu, J.; Li, C.-Y. Talanta 2018, 188, 691.

[37] Gong, S.; Hong, J.; Zhou, E.; Feng, G. Talanta 2019, 201, 40.

[38] Deng, Y.; Hong, J.; Zhou, E.; Feng, G. Dyes Pigm. 2019, 17, 107634

[39] Xu, Z. Y.; Yan, J. W.; Li, J.; Yao, P. F.; Tan, J. H.; Zhang, L. Tetrahedron Lett. 2016, 57, 2927.

[40] Das, B.; Lohar, S.; Patra, A.; Ahmmed, E.; Mandal, S. K.; Bhakta, J. N. New J. Chem. 2018, 42, 13497.

[41] Dhara, K.; Lohar, S.; Patra, A.; Roy, P.; Saha, S. K.; Sadhukhan, G. C. Anal. Chem. 2018, 90, 2933.

[42] Wang, Z.; Liu, C.; Wang, X.; Duan, Q.; Jia, P.; Zhu, H.; Li, Z.; Zhang, X.; Ren, X.; Zhu, B.; Sheng, W. Sens. Actuators, B 2019, $291,329$.

[43] Feng, W.; Feng, S.; Feng, G. Q. Anal. Chem. 2019, 91, 8602.

(Li, L.; Fan, Y.) 